\title{
Remarks on the Nyquist and Callen-Welton Theorems
}

\author{
S.A. Trigger ${ }^{1}$, G.J.F. van Heijst ${ }^{2}$, A.A. Rukhadze ${ }^{3}$, and P.P.J.M. Schram ${ }^{2}$ \\ 1 Joint Institute for High Temperatures, \\ Russian Academy of Sciences, 13/19, Izhorskaia Str., \\ Moscow127412, Russia; email: strig@gmx.net \\ 2 Eindhoven University of Technology, P.O. Box 513, \\ MB 5600 Eindhoven, The Netherlands and \\ 3 A.M. Prokhorov General Physics Institute Moscow, 119991, Russia
}

\begin{abstract}
The connection of the Callen-Welton and Nyquist fluctuation-dissipation relations is considered for plasma-like classical and quantum systems. The conditions for appearance of the dissipative parameters in the equilibrium current-current correlation function are investigated. The paper presents the arguments for the restrictions of the Nyquist theorem and against violation of the Callen-Welton theorem in the quantum case.
\end{abstract}

\section{INTRODUCTION}

The Callen-Welton theorem (CWT) is a general and rigorous result of statistical physics (see e.g. 1] ). Some ideas have been suggested 2, 3] to show that the existing form of CWT in the quantum case is not correct. One of the arguments is based on a consideration of the oscillatory electric circuit. As is well known, the classical Nyquist formula [4] for a random electromotive force (emf) $\varepsilon_{\omega}^{2}$ has the form:

$$
\varepsilon_{\omega}^{2}=2 T \operatorname{Re} Z(\omega)
$$

where $\mathrm{T}$ is the temperature in energetic units and $Z(\omega)$ is the impedance $Z(\omega)$ of a linear electric chain with the components arranged serially (for example). For a quasi-static field, changing slowly in time, $Z(\omega)$ is a function of the resistivity $R$, inductance $L$ and capacity $C$ for the electric RLC circuit (the speed of light is taken equal to unity $c=1$ ):

$$
Z=R-i\left(\omega L-\frac{1}{\omega C}\right)
$$


In the general case the function $Z(\omega)$ is the Fourier-component of the linear operator $\tilde{Z}$, which connects the functions $\varepsilon(t)$ and $J(t)$ in a linear relation:

$$
\varepsilon=\tilde{Z} J
$$

or in Fourier-components:

$$
\varepsilon_{\omega}=Z(\omega) J_{\omega} .
$$

For the current fluctuations one derives:

$$
\left|J_{\omega}\right|^{2}=2 T \frac{R^{\prime}}{R^{\prime 2}+\left(R^{\prime \prime}+\omega L-\frac{1}{\omega C}\right)^{2}},
$$

where $R^{\prime}$ and $R^{\prime \prime}$ are the real and imaginary parts of the resistivity $R(\omega)$, respectively. Let us now calculate the full average square of the magnetic energy of the current $L J^{2} / 2$ :

$$
\frac{L J^{2}}{2}=\frac{L}{2} \int_{-\infty}^{\infty} \frac{d \omega}{2 \pi}\left|J_{\omega}\right|^{2}=L T \int_{-\infty}^{\infty} \frac{d \omega}{2 \pi} \frac{R^{\prime}}{R^{\prime 2}+\left(R^{\prime \prime}+\omega L-\frac{1}{\omega C}\right)^{2}} .
$$

For the case of $\omega$-independent (and, therefore, real) resistivity $R_{0}$, which was considered in [2], Eq. (6) can be written in the form:

$$
\frac{L J^{2}}{2}=T \int_{-\infty}^{\infty} \frac{d \xi}{2 \pi} \frac{1}{1+\left(\xi-\frac{L}{C R_{0}^{2} \xi}\right)^{2}} .
$$

in which $\xi=L \omega / R_{0}$. The integral in Eq. (17) can be calculated analytically (or by use of, e.g., the mathematical program "Maple") and is equal to $1 / 2$. There is no dependence of the integral (7) on the parameter $L / C R_{0}^{2}$; therefore it is also independent of the dissipative parameter $R_{0}$. The analytical calculation of the integral (7) for the considered classical case of $\omega$-independent active resistivity $R_{0}$ is presented in the Appendix.

\section{FORMULATION OF THE PROBLEM, THE DISSIPATIVE AND PHYSICAL PARAMETERS}

The argumentation [2, 3] against the quantum generalization of the Nyquist theorem [4]

$$
\varepsilon_{\omega}^{2}=2 R T_{\omega} ; \quad T_{\omega}=\frac{\hbar \omega}{2} \operatorname{coth} \frac{\hbar \omega}{2 T}
$$

is essentially based on the notion, that the magnetic energy of the current (see Eq. (6)), as well as other correlation functions, calculated over the equilibrium distribution, cannot 
include the dissipative parameter [2, 3]. More concretely, this statement has been formulated as the requirement for the integral of the magnetic energy $L J^{2} / 2$ to be independent of the active resistivity $R_{0}$. This statement does not apply to the case of frequency-dependent $T_{\omega}$, when the magnetic energy is determined by an equation similar to Eq. (66). In the quantum case, however, the integral in (6) contains $T_{\omega}$, instead of the temperature $T$ in the classical case, and depends on $R_{0}$. According to [2, 3] it is the reason to reconsider the quantum Nyquist formula and the other quantum fluctuation-dissipation relations or theorems (FDT). We want to stress that in [5] the independence of the charge fluctuations in the electric circuit has been considered on the basis of thermodynamic requirements only for the quasi-static case $(\hbar \omega<T)$, e.g., in the classical case.

At first we have to consider and analyze the requirement of independence of the equilibrium correlation functions of the "dissipative parameters", as the basic one for reconsideration of the quantum FDT, in more detail. The simplest way to do that is to analyze microscopically the systems with Coulomb interaction, possessing a well-known Hamiltonian. It is evident, that the "dissipative parameters", such as, e.g., resistivity, themselves are functions of the fundamental non-dissipative parameters, namely, the mass $m_{\alpha}$, the charges $e_{\alpha}$, the particle density $n_{\alpha}$ (for the finite samples the characteristic sizes can appear) and also the temperature $T$. Therefore it is necessary to clarify this statement by considering the possible dependence of the equilibrium correlation functions on the dimensionless parameters. We can suggest, for example, that for plasma-like systems the integral over frequencies of the current-current correlation function $I \equiv J^{2}$ is of the form $I=T \Omega_{p}^{2} f(\Gamma, \lambda, Z)$, where $\Omega_{p}, Z, \Gamma \sim e^{2} n^{1 / 3} / T$ and $\lambda \sim \hbar^{2} n^{2 / 3} / m T$ are the plasma frequency, ion charge, interaction and degeneration parameters, respectively (the derivation of this statement is presented in Section III).

Instead of $\Gamma$ and $\lambda$ it is sometimes more convenient to use, as independent parameters, e.g., the parameters $\Gamma$ and $\mu=d / a_{0} \equiv \Gamma / \lambda$, where $d=n^{-1 / 3}$ and $a_{0}$ is the Bohr radius. In a finite system additional dimensionless parameters connected with the sizes of the sample can be present. In some particular case this integral can be independent of $\Gamma$ and has a form $I \simeq T \Omega_{p}^{2}$.

It seems natural to suggest that the statement on independence of the equilibrium correlation functions of the "dissipative parameters" in [2] can be formulated (in the case of the Coulomb systems) as the condition $I \simeq T \Omega_{p}^{2}$. 
Otherwise, if there is dependence on the other parameters mentioned above (at first on $\Gamma$, for almost classical plasma) the dissipation manifests itself in the correlation function. This conclusion follows, in particular, from the formula for the collision frequency of the Lorentz plasma $\nu_{0}=\pi \Omega_{p} \Gamma^{3 / 2} L / 8 \sqrt{2}$ of a weakly non-ideal plasma with $Z=1$. Here $L$ is the Coulomb logarithm, which for a weakly non-ideal $(\Gamma<1)$ classical plasmas can be taken (with logarithmic accuracy) as $L=\ln (1 / \Gamma)$. The frequency $\nu$ is, of course, the "dissipative" parameter in the sense of 2]. Therefore, it is natural to suggest that the dependence on "dissipative" parameters for $J^{2}$ means in fact the dependence on $\Gamma$. In any case, it is necessary to suggest which kind of dependence of the correlation functions, e.g. $J^{2}$, on the selected parameters can, in principle, exist. Only on this basis can be proved that the FDT are rigorously correct or that in some cases, e.g., for quantum systems an incorrectness exists.

We turn now to the Callen and Welton theorem (CWT) [6]. The CWT, strictly speaking, is formulated on the basis of statistical consideration of a system with a well- determined Hamiltonian and, therefore, can be applied to the Coulomb system with an arbitrary interaction parameter.

How to apply these results to the "macroscopic" electrodynamics, which are described in terms of macroscopical parameters as inductance and capacity? The answer is not quite clear, since an exact determination of correlation functions for these cases is lacking - as far as we know.

It should be noted that the work (per unit time) produced by emf $\varepsilon$ under the moving charges in the linear circuit is equal to the sum of Joule heating, change of the magnetic field and the energy of electric field in the capacitor [7]:

$$
\varepsilon J=R J^{2}+\frac{d}{d t}\left(\frac{L J^{2}}{2}+\frac{e^{2}}{2 C}\right) .
$$

This relation leads to the expression (2) for the frequency dependent impedance. At the same time the applicability of this representation is limited to the classical and quasi-static approximations. 


\section{FREQUENCY-DEPENDENT RESISTIVITY}

Nevertheless, let us assume that the representation (21) can be formally applied also in the quantum case. Let us also assume that some prohibition exists for the integrals of the correlation functions (in the case under consideration the integral of type (6)) to be dependent on the "dissipative" parameters (although, as was mentioned above, this requirement is not even clearly formulated). Under this assumption it seems that the arguments against the CWT in the quantum case in the approximation considered in [2] could be correct, because the $R_{0}$ dependence of the integral $I$ is absent in the classical case while it appears in the quantum case.

However, from our point of view, the arguments against the quantum generalization of CWT are not correct, because the approximation used in [2] is not general. The static approximation for resistivity (and, in general, for the impedance) is not correct for high frequencies, which are essential in the integral in Eq. (6). The dependence $R(\omega)$ can lead, as we will show below, to a dependence of the integral on the effective frequency of collisions if $L \neq 0$ and $C \neq \infty$, even in the classical case.

\section{A. The classical case for $L=0, C=\infty$.}

For the special case $L=0$ and $C=\infty$ we will show below, that: i) the case of $R$ being independent of $\omega$ is meaningless, because in this limit $J^{2} \sim 1 / L \rightarrow \infty$, whereas it must be finite, ii) if we use $R(\omega)$ we arrive at CWT for arbitrary interaction between the particles in both classical and quantum cases, iii) there is no fundamental prohibition for correlation functions, e.g. the integral $I$, to be dependent on the "dissipative parameters" (in the sense discussed above), although in the classical case this integral in fact is independent of it.

Now we turn to the main reasons showing that CWT is correct. It was already noted [8] that the impedance in quasi-static form, used above, is valid only for very low frequency (in the classical state as well as in the quantum case). In particular, for the frequencies $\hbar \omega / T \geq 1$, where the quantum CWT is applicable, the quasi-static form of the impedance cannot be used.

We will now show that even for the classical case the quasi-static approximation for the impedance $Z(\omega)$, described by Eq. (22) cannot be used and, therefore, the Nyquist formula is 
only an approximation, which is valid only for the quasi-static case of the small frequencies. At the same time the CWT is applicable for all frequencies and in this sense is a rigorous result for classical and quantum systems.

For this purpose we consider the specific circuit with $L=0$ and $C=\infty$ (capacitor with the distance between the plates $d=0$ ). Then, in the quasi-static approximation used in [2], $Z$ is equal to $R$ in this case and the integral is infinite if $R$ is $\omega$-independent.

In reality, for this type of circuit (the conductor with $L=0$ and $C=\infty$ ) we have:

$$
\frac{1}{R(\omega)}=\frac{\sigma(\omega) S}{l}
$$

where $\sigma(\omega)$ is the dynamic conductivity, $l$ and $S$ are the length and the area of the conductor section, respectively. Eq. (10) is the consequence of the relations $j(\omega)=\sigma(\omega) E(\omega)$ and $J=S j, \varepsilon(\omega)=l E(\omega)$ for the linear conductor.

For the simplest case under consideration the approximate expression for the conductivity can be written in the Drude-Lorentz form:

$$
\sigma_{D L}(\omega)=\frac{i n e^{2}}{m(\omega+i \nu)}
$$

where $\nu$ is the effective, $\omega$-independent, collision frequency of the particles, which transfer the current. Substituting Eqs. (101),(11) in Eq. (7) with $L=0$ and $C=\infty$ we arrive at the integral:

$$
J^{2}=\int_{-\infty}^{\infty} \frac{d \omega}{2 \pi}\left|J_{\omega}\right|^{2}=2 D T \int_{-\infty}^{\infty} \frac{d \omega}{2 \pi} \operatorname{Re} \sigma(\omega) .
$$

For the Drude-Lorentz conductivity we find from Eq. (12)

$$
J^{2}=2 D T \int_{-\infty}^{\infty} \frac{d \omega}{2 \pi} \operatorname{Re} \sigma_{D L}(\omega)=\frac{2 D T n e^{2}}{m} \int_{-\infty}^{\infty} \frac{d \omega}{2 \pi} \frac{\nu}{\omega^{2}+\nu^{2}} .
$$

The result of the integration is, naturally, finite and equals $D T n e^{2} / m \equiv D T \Omega_{p e}^{2} / 4 \pi$, where $D \equiv S / l$. Apparently, this result does not involve the dissipative parameter $\nu$. This implies that $J^{2}$ does not involve the interaction parameter $\Gamma$ (we can consider this fact as a specific condition for the non-dissipative character of correlation functions, see above).

It is necessary to stress, that in Eq. (12) we used the so-called internal conductivity (11), which we choose in the Drude-Lorentz approximation. This conductivity is the response function for the self-consistent electrical field in Coulomb systems. The general relation 
between the internal conductivity $\sigma(\omega)$ and the external conductivity $\sigma_{e x}(\omega)$ (the response on an external field applied to the system) has the form [9]:

$$
\sigma(\omega)=\frac{\sigma_{e x}(\omega)}{1-\frac{4 \pi i \sigma_{e x}(\omega)}{\omega}},
$$

It is easy to show by straight calculation that the integral (12) has the same value for $\sigma(\omega)$ and $\sigma_{e x}(\omega)$ if we use the Drude-Lorentz approximation. In the general case for the exact (non-approximative) expressions for conductivities it is also true [10], as can be shown by using of the Kramers-Kronig relations for $\sigma_{e x}(\omega)$ and $\sigma(\omega)$. The problem of the validity of the Kramers-Kronig relations for conductivities has been investigated by Kirzhnits in [11]. In the long-wavelength limit not only $\sigma_{e x}$, but also the dielectric function $\varepsilon(\omega)$ and therefore $\sigma(\omega)$ satisfy the Kramers-Kronig relations (w.r.t. the external conductivity this statement is valid also for finite values of the wave vector $k$ ). The resistivity, present in the relations (22),(12), is the internal one, because only the internal conductivity $\sigma$ has a finite limit for $\omega \rightarrow 0$ and provides a finite value of $R^{\prime}(\omega \rightarrow 0) \equiv R e R(\omega \rightarrow 0)$. The external coductivity in the limit $\omega \rightarrow 0$ tends to zero as $\omega^{2}$ (see, e.g., 12]).

By applying the sum rule [13] for the external conductivity of a system of charges with arbitrary strong interaction between the particles:

$$
\int_{-\infty}^{\infty} \frac{d \omega}{\pi} \operatorname{Re} \sigma_{e x}(\omega)=\sum_{r} \frac{e_{r}^{2} n_{r}}{m_{r}}
$$

with $r$ the index of the species of particles, we arrive at a general relation similar to Eq. (12), but without any assumption about the concrete form of the conductivity for the classical system:

$$
J^{2}=D T \int_{-\infty}^{\infty} \frac{d \omega}{\pi} \operatorname{Re} \sigma_{e x}(\omega)=D T \sum_{r} \frac{e_{r}^{2} n_{r}}{m_{r}} .
$$

The additional assumption that has been adapted to establish Eq. (15) is isotropy of the system under consideration. This means, in particular, that the conductivity tensor is diagonal with equal components. According to the statements considered above, Eqs. (15), (16) are also satisfied if instead of $\sigma_{e x}$ we write $\sigma$.

As is evident from the general point of view, the current-current correlation function in the classical case is independent of the "dissipative" parameters. 


\section{B. The case of a classical system with $L \neq 0, C \neq \infty$.}

Let us now consider, as an example of the classical case, the integral (6) with $L \neq 0$ and $C \neq \infty$. We assume that the terms related with the inductance and capacitor in Eq. (2) for the impedance are unchanged. For the Drude-Lorentz internal resistivity with constant $\nu(\omega)=\nu$ the real and imaginary parts of the resistivity are equal to $\operatorname{Re} R_{\text {in }}(\omega)=R_{0} \equiv$ $m \nu / D n e^{2}$ and $\operatorname{Im}_{\text {in }}(\omega)=-m \omega / D n e^{2}$, respectively, where $R e R_{\text {in }}(\omega)$ is independent of $\omega$. For the external resistivity in Drude-Lorentz approximation we obtain $\operatorname{Re} R(\omega)=R_{0} \equiv$ $m \nu / D n e^{2}$ and $\operatorname{Im} R(\omega)=-m\left(\omega^{2}-\Omega_{p}^{2}\right) / D \omega n e^{2}=-R_{0}\left(\omega^{2}-\Omega_{p}^{2}\right) / \nu \omega$.

Then we return to Eq. (77) and to the equality $L J^{2}=T L / \tilde{L}$ with the effective $\tilde{L}=$ $L-\left(m / D n e^{2}\right)$. It is evident that the condition $\tilde{L}>0$ must be fulfilled, which demonstrates the limited applicability of the above formulated assumption. However, if we take into account that the expression (15) is the result for the thermodynamic limit, whereas the Nyquist formula is written for finite samples, we have to consider large values of $D$ and on this basis we can suggest $\tilde{L}>0$.

In general, we have to stress the difference between FD relations for finite systems and for systems in the thermodynamic limit. Consideration of the case $L \neq 0$ and $C \neq \infty$ has conventional character, because the value $\tilde{L}$ can change sign, although the value $J^{2}$ must be positive according to definition. This means that representation (6) has, strictly speaking, to be additionally modified for the case $L \neq 0$ and $C \neq \infty$, when we take into account the $\omega$-dependence for the resistivity $R$.

Nevertheless, it is easy to see, even for the restricted modification of Eq. (6), when we use the Drude-Lorentz conductivity with $\omega$-dependent $\nu(\omega)$ the resistivity $R_{0} \rightarrow R_{0} \theta(\omega)$ (in general, $\theta(\omega)$ has real and imaginary parts $\theta^{\prime}(\omega)$ and $\left.\theta^{\prime \prime}(\omega)\right)$ and the statement about the energy $L J^{2}$ being independent of dissipative parameter is not valid. To show this, let us consider the conductivity of the Lorentz classical plasma system:

$$
\sigma(\omega)=\frac{i e^{2}}{3 T} \int \frac{v^{2} f_{0}(p)}{\omega+i \nu(v)} d^{3} p
$$

where $f_{0}(p)$ is the Maxwellian distribution, normalized to the electron density $n_{e}$, and $\nu(v)=$ $4 \pi Z e^{4} n_{e} L / m^{2} v^{3}$ is the velocity-dependent effective collision frequency for charged particles. In this approach we use the notation $R_{0} \equiv m \nu_{0} / D n e^{2}$. The functions $\theta^{\prime}(\omega)$ and $\theta^{\prime \prime}(\omega)$ can be found by using the relation $R=1 / D \sigma$, where $\sigma$ is determined by (17). As follows on 
the basis of Eq. (177), the function $\theta^{\prime} \neq$ const and $\theta^{\prime \prime}$ is a nonlinear function of $\omega / \nu_{0}$. In particular, in the high-frequency region $\left(\omega>\nu_{0}\right)$ the functions $\theta^{\prime}(\omega)$ and $\theta^{\prime \prime}(\omega)$ are equal to:

$$
\begin{gathered}
\theta^{\prime}(\omega)=\frac{32}{3 \pi} \frac{\omega^{2}}{\omega^{2}+\nu_{1}^{2}}, \\
\theta^{\prime \prime}(\omega)=-\frac{\omega^{3}}{\nu_{0}\left(\omega^{2}+\nu_{1}^{2}\right)},
\end{gathered}
$$

where $\nu_{1}=32 \nu_{0} / 3 \pi$. This means that the integral (6) cannot be represented in a form similar to (77) with some effective constants $L$ and $C$ and depends on the "dissipative" parameter $R_{0}$.

Therefore in the case of a system with $L \neq 0$ and $C \neq \infty$ the statement about independence of the correlation function of "dissipative parameters" is not true even in the classical case, at least for a consideration based on the same dependence $Z(\omega)$ on $L$ and $C$ as in Eq. (2).

\section{The quantum system with $L=0$ and $C=\infty$, Callen-Welton relation.}

We now turn to the quantum case with $L=0$ and $C=\infty$. The sum rule (15) is valid in the quantum case as well [13]. At the same time the correlation function $J^{2}$ has to be written (see, e.g. [13]) as:

$$
J^{2}=D \int_{-\infty}^{\infty} \frac{d \omega}{\pi} T_{\omega} \operatorname{Re} \sigma(\omega) .
$$

This result can be easily obtained from the general exact CWT for the density current correlation function:

$$
<j_{i}(\mathbf{r}, t) j_{k}\left(\mathbf{r}^{\prime}, t^{\prime}\right)>=\frac{\hbar \omega}{4 \pi} \operatorname{coth}\left(\frac{\hbar \omega}{2 T}\right)\left[\sigma_{i k}(\omega)+\sigma_{k i}^{*}(\omega)\right] \delta\left(\mathbf{r}-\mathbf{r}^{\prime}\right)
$$

where $\sigma_{i k}$ is a component of the tensorial conductivity and the asterisk denotes the complex conjugate by integration over $\mathbf{r}$ and $\mathbf{r}^{\prime}$ in the volume of a linear conductor $V=l S$ and the suggestion that in this volume the conductivity is the same as in the thermodynamic limit. Under this suggestion the result coincides with the Nyquist relation in the form (201), where conductivity is an exact function of the frequency $\omega$.

There exist no arguments for independence of this integral of the parameter $\Gamma$. Therefore this integral depends on "dissipative parameters". Let us demonstrate this fact in the 
example of the expansion of $J^{2}$ in the parameter $\hbar \omega / T$. In this case the Eq. (20) can be rewritten approximately in terms of the zeroth and second momenta of the conductivity:

$$
J^{2} \simeq D \int_{-\infty}^{\infty} \frac{d \omega}{\pi} T_{\omega} \operatorname{Re} \sigma(\omega) \simeq \frac{D T}{\pi}\left(\mu_{0}+\frac{\hbar^{2} \mu_{2}}{12 T^{2}}\right)
$$

where

$$
\mu_{2 n} \equiv \int_{-\infty}^{\infty} d \omega \omega^{2 n} \operatorname{Re} \sigma(\omega)
$$

The term with $\mu_{0}=\Omega_{p}^{2} / 4$ leads to the classical value of $J^{2}$ (see Eq. (20)). The second moment of the external conductivity has been calculated in 10] and can be expressed in terms of the value of the electron-ion equilibrium correlation function $g_{e i}(\mathbf{r})$ at $r=0$. It can be connected with the second moment of the internal conductivity by use of the asymptotic expansions for the Kramers-Kronig formulas. For the case $Z=1$ the second moment of the internal conductivity equals:

$$
\mu_{2}=\frac{\Omega_{p e}^{4} g_{e i}(0)}{24}
$$

The final expression for $J^{2}$ following from the relations mentioned above is:

$$
J^{2} \simeq \frac{D T \Omega_{p e}^{2}}{4 \pi}\left(1+\frac{\hbar^{2} \Omega_{p e}^{2} g_{e i}(0)}{6 T^{2}}\right)
$$

Naturally the function $g_{e i}(\mathbf{r})$, as well as it value in the point $r=0$, is a function of the parameter $\Gamma$. To calculate $g_{e i}(0)$ for a purely Coulomb system the interaction and quantum effects in $g_{e i}$ also have to be taken into account, in particular to avoid the divergence $g_{e i}(0)$ for small distances. For example, for a weak plasma interaction $\Gamma \ll 1$ the pair correlation function can be written $(\mathrm{Z}=1)$ as $[14]$ :

$$
g_{e i}(r)=S_{e i}^{(2)}(r) \exp \left[\frac{e^{2}}{4 \pi T r}\left(1-e^{-\kappa r}\right)\right],
$$

where $\kappa^{-1}=\sqrt{T / 4 \pi e^{2} n}$ is the Debye radius (with the density $n=n_{e}+n_{i}$ ) and $S_{e i}^{(2)}(r)$ is the pair electron-ion Slater sum, which takes into account the quantum effects. The value $g_{e i}(0)$ for the approximation (201) )equals:

$$
g_{e i}(0)=S_{e i}^{(2)}(0) \exp \left(\frac{\Gamma^{3 / 2}}{\sqrt{4 \pi}}\right)
$$


The Slater sum $S_{e i}^{(2)}(0)$ is a function of the parameter $\zeta=e^{2} / 4 \pi T \lambda_{e i}$, where $\lambda_{e i}=\hbar / \sqrt{2 m T}$ and $m$ are De Broglie wavelength and the reduced electro-ion mass respectively. This function has a finite value [14] for all values of the parameter $\lambda_{e i}$, except $\lambda_{e i}=0$.

Therefore $J^{2}$, even in the simple approximations used above, depends on the "dissipative" parameter $\Gamma$. The dependence of the current-current equilibrium correlation functions on the "dissipative" parameters is not in contradiction with any fundamental principles of quantum statistical theory.

\section{CONCLUSIONS.}

The main conclusion of the present paper is the statement that the quasi-static approximation for the impedance in the form used in [2, 4] is not allowed when considering the opportunity for violation of the general quantum CWT. Therefore, presently there are no arguments to discuss violation of the CWT in the quantum case on this basis. As it is, strictly speaking, the CWT is proved only for the case of a conductor with $L=0$ and $C=\infty$ and for an infinite medium, when the Hamiltonian description of the system is welldetermined. For this case we have shown the way to connect the macroscopical model of the Nyquist fluctuation-dissipation relation with the microscopic derivation of the CWT by introduction of the frequency dependent resistivity in the Nyquist model. We also demonstrated the absence at this time of a clear way for generalization of this relation for the case $L \neq 0$ and $C \neq \infty$, in spite of some attempts to do that [3]. The important question about determination of the "dissipative" parameters has been discussed above for plasmalike systems and connected, in particular cases considered above, with the $\Gamma$-dependence of the current-current correlation function. In general, a more fundamental determination of the dissipative parameters has to be formulated.

In the framework of the kinetic equations, the dissipative parameters are connected with the respective effective collision frequencies. For the problem of FD relations the approach of kinetic equations is not enough, because the results have to be found for arbitrarily strong interaction between the particles. In this case the determination of the dissipative parameters is based, in general, on the imaginary parts of the poles of the analytical continuation of the temperature Green functions [15]. In the particular cases of weak or short-range interactions between the particles these poles lead to the same results as the kinetic equations approach. 
The problem of the validity of summation of the rows of the perturbation theory for the Green functions, as well as the problem of irreversible behavior of physical systems due to damping, connected with these poles, have a fundamental character and still have not been completely clarified. Nevertheless, we trust that the general results, connected with dissipation based on the Green function approach are applicable to obtain the fluctuationdissipation relations for systems with well-defined Hamiltonian. Of cause the $\Gamma$ - dependence of the current-current correlation function, which we consider as a manifestation of the dependence on "dissipative parameters", can be related in fact not only with a shifting of the imaginary part of the poles of the Green functions, but also with a shifting of the real parts of these poles. Nevertheless we do not see the opportunity to distinguish in the exact final result for CWT the "dissipative" and "non-dissipative" dependence on the parameter $\Gamma$.

The sense of the consideration, suggested by Yu.L. Klimontovich [2] is, from our point of view, not about incorrectness of the CWT, but about limited applicability of the Nyquist formula in the approximation of constant $R$. This limitation follows, at first, as was shown above, from the quasi-static character of the impedance in the form (2). It is evident not only in the quantum, but also in the classical case. At the moment there is no clear basis for a general and rigorous consideration of the electrical circuit with finite $L \neq 0$ and $C \neq \infty$ in the quantum and even in the classical case, except the model of quasi-static approximation for the impedance, which is applicable nevertheless to many experimental situations.

\section{APPENDIX.}

The integral $I$ in Eq. (7),

$$
I=\frac{1}{\pi} \int_{-\infty}^{\infty} d \xi \frac{1}{1+\left(\xi-\frac{L}{C R_{0}^{2} \xi}\right)^{2}}
$$

can be calculated analytically. According to the Maple calculation program this integral equals one. We can rewrite the integral (28) in the form

$$
I=\frac{1}{\pi} \int_{-\infty}^{\infty} d \xi \frac{\xi^{2}}{\xi^{2}+\left(\xi^{2}-\lambda\right)^{2}} .
$$


with $\lambda \equiv L / C R_{0}^{2}>0$. The denominator is equal to $\left(\xi^{2}-\xi_{1}^{2}\right)\left(\xi^{2}-\xi_{2}^{2}\right)$, where the roots are:

$$
\xi_{1}^{2}=\frac{1}{2}[-(1-2 \lambda)+\sqrt{(1-4 \lambda)}], \xi_{2}^{2}=\frac{1}{2}[-(1-2 \lambda)-\sqrt{(1-4 \lambda)}]
$$

The integral (7) can be split into two integrals $I=I_{1}+I_{2}$, with

$$
I_{1}=\frac{1}{\pi \sqrt{(1-4 \lambda)}} \int_{-\infty}^{\infty} d \xi \frac{\xi_{1}^{2}}{\left(\xi^{2}-\xi_{1}^{2}\right)}, I_{2}=-\frac{1}{\pi \sqrt{(1-4 \lambda)}} \int_{-\infty}^{\infty} d \xi \frac{\xi_{2}^{2}}{\left(\xi^{2}-\xi_{2}^{2}\right)}
$$

Let us consider the particular case $1-4 \lambda>0$. It is easy to see that the roots $\xi_{1}^{2}, \xi_{2}^{2}$ in this case are real and negative. Therefore the integrals are equal to:

$$
I_{1}=-\frac{\sqrt{\left|\xi_{1}^{2}\right|}}{\sqrt{(1-4 \lambda)}}, I_{2}=\frac{\sqrt{\left|\xi_{2}^{2}\right|}}{\sqrt{(1-4 \lambda)}} .
$$

Because $\left|\xi_{2}^{2}\right|>\left|\xi_{1}^{2}\right|$ the sum $I=I_{1}+I_{2}>0$ and equals unity. To show this it suffices to take $I^{2}$ and to use Eq. (32). The same is true for an arbitrary value of $\lambda$.

\section{Acknowledgment}

Authors are gateful to W. Ebeling, A.M. Ignatov, N.I. Klyuchnikov, and I.M. Tkachenko for valuable discussions of the various problems, reflected in this work, which has been submitted in Physica A (8 March 2006).

[1] L.D. Landau, E.M.Lifshitz, Statistical Physics, "Science" Publishing, Moscow, 1976.

[2] Yu.L. Klimontovich, Uspekhi Fiz. Nauk, 151, 2, 309 (1987). Private communications (19992001).

[3] B.A. Veklenko, Yu.B. Scherkunov, Applied Physics (in Russian), 4, 5 (2004).

[4] H. Nyquist, Phys. Rev., 32, 110 (1928).

[5] G.S. Gorelik, Uspekhi Fiz. Nauk, XLIV, 1, 33 (1951).

[6] H.B. Callen, Th.A. Welton, Phys. Rev., 83, 34 (1951).

[7] L.D. Landau, E.M. Lifshitz, Electrodynamics of the Continuous Media, Fizmatgiz, Moscow, 1959.

[8] S.A. Trigger, Meetings with Yu.L. Klimontovich, Condensed Matter Physics, 7, N 3 (39), 443 (2004); DAMU-Hefte, 1 (2005) 56 (Berlin). 
[9] A.I.Akhiezer, S.V. Peletminski, Methods of Statistical Physics, "Science" Publishing, Moscow, 1977.

[10] V.M. Adamyan, I.M. Tkachenko, Contr. Pl. Phys. 43, 252 (2003).

[11] D.A. Kirzhnits, Uspekhi Fiz. Nauk, 119, 2, 357 (1976).

[12] D. Pines, P. Nozieres, The Theory of Quantum Liquids, v.I, W.A. Benjamin, INC. New York - Amsterdam, 1966.

[13] R. Kubo, J. Phys. Soc. Japan, 12, 570 (1957).

[14] W.D. Kraeft, D. Kremp, W.Ebeling, G. Roepke, Quantum Statistics of Charged Particle Systems, Akademie Verlag, Berlin, 1986.

[15] A.A. Abrikosov, L.P. Gor'kov, I.E. Dzyaloshinskii, Methods of Quantum Field Theory in Statistical Physics, Fiz-Mat. Publishing, Moscow, 1962. 